COLON CANCER

\title{
Sporadic duodenal adenoma is associated with colorectal neoplasia
}

\author{
M A Murray, M J Zimmerman, H C Ee
}

See end of article for authors' affiliations

.....................

Correspondence to:

Dr H C Ee, Department of

Gastroenterology and

Hepatology, Sir Charles

Gairdner Hospital,

Nedlands, Western

Australia, Australia;

hooi.ee@health.wa.gov.au

Accepted for publication

3 September 2003
Objective: The objective of this study was to assess the association between colorectal neoplasia and sporadic duodenal adenoma.

Methods: A retrospective case control study was conducted using the databases of two major teaching hospitals in Western Australia. The frequency of colorectal neoplasia in patients with sporadic duodenal adenomas was compared with that in a control group of patients presenting for endoscopies. The frequency of colorectal cancer in duodenal adenoma patients was also compared with the population incidence.

Results: Of 56 sporadic duodenal adenoma patients, 34 (61\%) had been colonoscoped. When comparing the findings between patients with sporadic duodenal adenoma and an endoscoped control group, all colorectal neoplasias were significantly more common in the duodenal adenoma group $156 \%$ v $33 \%$; odds ratio (OR) 2.4 (95\% confidence intervals (CI) 1.1-5.4)). Although finding either advanced colorectal adenoma or cancer was also more common in duodenal adenoma patients $138 \% \mathrm{v} 19 \%$; OR $2.3195 \% \mathrm{Cl}$ 1.0-5.2)), as was finding colorectal cancer alone (21\% v 8\%; OR $3.0(95 \% \mathrm{Cl} 1.0-9.1))$, the results were not statistically significant. However, the incidence of colorectal cancer was much greater in duodenal adenoma patients than in the population $(p<0.001)$.

Conclusions: Sporadic duodenal adenoma has a clinically important association with colorectal neoplasia. Thus patients with duodenal adenomas should undergo colonoscopy to detect colorectal neoplasia.
$\mathrm{S}$ poradic duodenal adenoma is an uncommon finding. In a large series from Germany, only $6.9 \%$ of 378 duodenal polyps found at 25000 upper gastrointestinal endoscopies were adenomatous. ${ }^{1}$ Another series similarly found that only $0.4 \%$ of 584 endoscopy patients had duodenal polyps, of which $7 \%$ were adenomatous. ${ }^{2}$ Most adenomas were found incidentally at endoscopy but occasionally caused bleeding or obstruction of the duodenum or ampulla of Vater. The distribution of small bowel adenomas shows a predominance at the ampulla and periampullary region, with decreased numbers proximally in the duodenum and distally in the small bowel, with a small peak in the distal ileum. ${ }^{3}$

Adenomas of the small and large intestine are neoplasiasthat is, they lie along a multistep pathway to carcinogenesis-effected by numerous genetic and epigenetic events. ${ }^{3-6}$ Curiously however, despite numerous phenotypic similarities between small and large intestinal epithelia, including a very high cellular turnover, small intestinal neoplasia is very rare compared with its colorectal counterpart. ${ }^{7}$ None the less, small intestinal carcinoma is associated with colorectal carcinoma and vice versa, ${ }^{8}$ but not gastric or oesophageal carcinoma. ${ }^{9}$

The extent to which a duodenal adenoma is associated with colorectal neoplasia is not well described. A previous clinicopathological study described 21 cases of duodenal adenomas, of which 11 underwent colonoscopy. ${ }^{10}$ Four of these 11 cases were classified as having familial adenomatous polyposis. Of the remaining seven cases of sporadic duodenal adenomas, four $(57 \%)$ were found to have colorectal neoplasia.

We aimed to see if finding a sporadic duodenal adenoma was a sign of associated colorectal neoplasia. Identifying such an association would allow earlier detection of colorectal neoplasia, as well as providing some insight into duodenal and colorectal carcinogenetic pathways. We determined the frequency of colorectal neoplasia in a relatively large group of patients with sporadic duodenal adenomas and compared this with a matched control group of symptomatic patients presenting for endoscopic procedures. Furthermore, we also compared the frequency of colorectal cancer in patients with sporadic duodenal adenomas with the incidence of colorectal cancer in the population.

\section{PATIENTS AND METHODS}

Patients with a diagnosis of duodenal adenoma were identified using the pathology coding databases of the two major metropolitan university teaching hospitals (Sir Charles Gairdner Hospital and Royal Perth Hospital) in Perth, Western Australia, during the period 1992-2002. Endoscopic reports of the identified patients were retrieved to confirm the location of the adenomas and to complete the descriptive record of the cases. Patients with, or belonging to, familial adenomatous polyposis (FAP) and hereditary nonpolyposis colorectal cancer (HNPCC) kindreds were excluded. Clinical records for identified patients were examined for associated synchronous or metachronous diagnoses of colorectal neoplasia. Only the most histologically advanced colorectal lesion was recorded for each patient.

Duodenal adenoma location, size, and histology were recorded. Similar details were recorded for colorectal neoplasms, when identified. Patients with multiple colorectal neoplasms were categorised according to the most advanced lesion found. Advanced adenomas (duodenal and colorectal) were defined as having a size $\geqslant 10 \mathrm{~mm}$, the presence of a villous component, or high grade dysplasia, ${ }^{11}{ }^{12}$ Indications

\footnotetext{
Abbreviations: $\mathrm{Cl}$, confidence intervals; $\mathrm{FAP}$, familial adenomatous polyposis; HNPCC, hereditary non-polyposis colorectal cancer; OR, odds ratio
} 
for upper gastrointestinal endoscopy and colonoscopy were recorded.

Findings in patients with duodenal adenomas were compared with: (1) endoscoped control patients, who were randomly selected age and sex matched patients presenting to Sir Charles Gairdner Hospital for both upper endoscopy and colonoscopy; and (2) population statistics, in which the incidence of colorectal cancer in the age and sex matched population was obtained from published Australian statistics. These statistics are compiled by the Australian Institute of Health and Welfare and the Australian Association of Cancer Registries and are available on the internet (http:// www.aihw.gov.au/publications/can/ca98). ${ }^{13}$

Conditional logistic regression derived odds ratios (OR) and $95 \%$ confidence intervals (CI) were used to compare the rates of colorectal neoplasia in the sporadic duodenal adenoma group with those of the endoscoped controls, and the $\chi^{2}$ test with continuity correction was used to compare duodenal adenoma patients with the population.

\section{RESULTS}

From January 1992 until June 2002 (inclusive), 57618 upper gastrointestinal endoscopies were performed on 39784 patients. A total of 100 patients were identified on the basis of the pathology code for duodenal adenoma. All identified specimens were obtained endoscopically. However, 44 patients were excluded because of FAP (24), HNPCC (1), invasive carcinoma (10), and other diagnoses (9).

\section{Sporadic duodenal adenomas}

Of the remaining 56 patients with sporadic duodenal adenomas, 31 were males and 25 were females. Mean age of the patients was 67 years (males 71 years; females 62 years), with a range of 38-91 years. Indications for upper gastrointestinal endoscopy are shown in table 1 , and were predominantly for anaemia, abdominal pain, and indications requiring endoscopic retrograde cholangiopancreatography.

A single duodenal adenoma was identified in each of the 56 patients. Adenomas were located in the duodenal cap (6 $(11 \%))$, second part of the duodenum (32 (57\%)), or ampullary region $(18(32 \%))$. Adenoma size was accurately reported in 44 patients, with a mean and median of $15 \mathrm{~mm}$ (range 3-70 mm). Large adenomas ( $\geqslant 10 \mathrm{~mm}$ ) were found in at least 28 patients $(50 \%)$. In 10 of 12 patients where adenoma size was not precisely reported, the ampulla was involved. Villosity was reported in part or in toto in 21 adenomas $(38 \%)$. High grade dysplasia was reported in two adenomas (4\%). Thus in at least 34 patients $(61 \%)$ the adenomas were advanced. There were no statistically significant relationships between adenoma location, size, or histology within this study group.

\section{Colorectal neoplasia associated with duodenal adenomas}

Of the 56 patients with duodenal adenomas, 34 (61\%) had at least one colonoscopy. Colorectal neoplasia was found in 19 of these 34 patients $(56 \%)$. Colorectal cancer was found in seven patients $(21 \%)$, advanced colorectal adenoma was found in six patients (18\%), and non-advanced colorectal adenoma was found in the remaining six patients $(18 \%)$. Thus 13 of the 34 patients with colorectal neoplasia (38\%) had either colorectal cancer or an advanced adenoma. Of the seven patients with colorectal cancer, eight cancers were actually found, with four located in the caecum, one in the ascending colon, two in the sigmoid, and one in the rectum. There was no significant relationship between different locations of the duodenal adenomas and the presence or nature of colorectal neoplasia. There was also no significant relationship between whether a duodenal adenoma was advanced or non-advanced and the presence or nature of colorectal neoplasia.

To confer clinical perspective to our identified rate of colorectal neoplasia in patients with sporadic duodenal adenomas, we compared each case with three randomly selected age and sex matched controls, consisting of symptomatic patients undergoing endoscopy and colonoscopy. Control patients were also obtained from our endoscopic database. Of 102 endoscoped control patients, 34 were found to have colorectal neoplasia $(33 \%)$. This consisted of colorectal cancer in eight patients $(8 \%)$, advanced adenomas in 11 patients $(11 \%)$, and non-advanced adenomas in 15 patients (15\%). In all, 19 patients (19\%) had either colorectal cancer or an advanced colorectal adenoma. Importantly, patients with sporadic duodenal adenomas, when compared with endoscoped controls, had a significantly greater risk of associated colorectal neoplasia (55\% v 33\%; p = 0. 03; OR 2.4 (95\% CI 1.1-5.4)). Although duodenal adenoma patients when compared with endoscoped controls were more likely to have advanced colorectal neoplasia-that is, advanced adenoma or cancer (38\% v 19\%; p = 0.05; OR 2.3 (95\% CI 1.0$5.2)$ ) and colorectal cancer alone ( $21 \% v 8 \%$; $=0.05$; OR 3.0 (95\% CI 1.0-9.1)) - the results were not statistically significant because the confidence intervals included 1.0 (tables 2 and 3 ).

\section{Colorectal cancer in patients with duodenal adenoma compared with the population}

We further compared the risk of colorectal cancer between patients with sporadic duodenal adenoma and that of age and sex matched population controls, based on Australian cancer statistics. ${ }^{13}$ The cumulative incidence in 34 matched population controls yielded an expected finding of 0.1 cancers $(0.3 \%)$ in this group. This contrasts with the observed findings of seven cancers in 34 patients with duodenal

\begin{tabular}{|c|c|c|}
\hline Indication & $n$ & Details \\
\hline Pain & 14 & Reflux (5), dyspepsia (5), epigastric pain (4) \\
\hline Anaemia & 13 & Anaemia or iron deficiency (13) \\
\hline Melaena & 5 & \\
\hline ERCP & 14 & Stones (6), abnormal LFT (2), pancreatitis (3), jaundice (3) \\
\hline Abnormal $x$ ray & 2 & Duodenal lesion (1), gastric ulcer (1) \\
\hline Dysphagia & 3 & \\
\hline Diarrhoea & 1 & \\
\hline Nausea and vomiting & 1 & \\
\hline Not recorded & 3 & \\
\hline Total & 56 & \\
\hline
\end{tabular}


Table 2 Proportion of duodenal adenoma patients and endoscoped controls with identified colorectal neoplasia

\begin{tabular}{llll}
\hline Colorectal neoplasia found* & $\begin{array}{l}\text { Duodenal adenoma } \\
(\mathbf{n}=34)\end{array}$ & $\begin{array}{l}\text { Controls } \\
(\mathbf{n = 1 0 2 )}\end{array}$ & p Value \\
\hline All colorectal neoplasia & $19(56 \%)$ & $34(33 \%)$ & 0.03 \\
Cancer or advanced adenoma & $13(38 \%)$ & $19(19 \%)$ & 0.05 (NS, see text) \\
Cancer & $7(21 \%)$ & $8(8 \%)$ & 0.05 (NS, see text) \\
Advanced adenoma & $6(18 \%)$ & $11(11 \%)$ & $0.44 \mathrm{NS}$ \\
Non-advanced adenoma & $6(18 \%)$ & $15(26 \%)$ & $0.51 \mathrm{NS}$ \\
\hline *Patients were classified according to the most advanced lesion found at colonoscopy.
\end{tabular}

adenomas $(21 \%)$ and was highly statistically significant $(\mathrm{p}<0.001)$.

\section{Indications for colonoscopy (table 4)}

Of the 34 patients with duodenal adenomas who were colonoscoped, $15(44 \%)$ of the procedures were for blood loss indications-namely, anaemia (13) or frank bleeding (2). This was not significantly different from 52 of 102 patients $(51 \%)$ in the endoscoped control group $(p=0.49)$. Importantly, 15 patients underwent colonoscopy purely on the basis of identification of a duodenal adenoma and seven $(47 \%)$ of these were found to harbour colorectal neoplasia. One patient was found to have colorectal cancer five years after identification of an ampullary villous adenoma, and another patient was found to have a sigmoid carcinoma having presented with rectal bleeding three months after incidental identification of a sporadic duodenal advanced adenoma.

\section{DISCUSSION}

In this study we have demonstrated a strong association between sporadic duodenal adenomas and colorectal neoplasia. When compared with an age and sex matched group of symptomatic patients undergoing upper endoscopy and colonoscopy, the yield of colorectal neoplasia was significantly greater for patients with duodenal adenoma (55\% $\mathrm{v}$ $33 \% ; \mathrm{p}=0.03$ ). Advanced colorectal adenoma or cancer was also more common among patients with duodenal adenomas $(38 \% \vee 19 \%$; p $=0.05$; OR $2.3(95 \%$ CI $1.0-5.2))$, as was the finding of cancer alone $(21 \% v 8 \%$; p $=0.05$; OR 3.0 (95\% CI 1.0-9.1)), although by definition a confidence interval including 1.0 is not statistically significant. The odds ratios of 2.3 and 3.0 are clinically important in magnitude, but the wide confidence intervals (1.0-5.2 and 1.0-9.1, respectively) suggest that our series lacked power to establish statistical significance. A larger series, albeit of a rare condition, may resolve the issue more precisely. It is none the less noteworthy that being symptomatic, the control patients already have a much higher incidence of colorectal neoplasia than the general population. This was confirmed by our finding that the rate of colorectal cancer in patients with duodenal adenomas was substantially greater than the age and sex matched cumulative incidence rates, as determined by population statistics $(21 \% \vee 0.3 \% ; \mathrm{p}<0.001)$.

The present study is the largest of its type, involving the highest number of sporadic duodenal adenomas. Our findings add to those of a previous small study identifying colorectal neoplasia in four of seven patients (57\%) with sporadic duodenal adenomas. ${ }^{10}$ Previous studies have shown that the diagnosis of small intestinal carcinoma increases the risk of finding colorectal cancer but not other gastrointestinal tract malignancy. ${ }^{89}$ For instance, Neugut and Santos ${ }^{8}$ revealed that after a diagnosis of primary adenocarcinoma of the small intestine, the relative risk of finding a primary adenocarcinoma of the colon or rectum was 5.0 (95\% CI $2.3-$ 9.4) in men and 3.7 (95\% CI 1.3-8.0) in women compared with expected numbers derived from population based tumour registries. We excluded duodenal or ampullary carcinomas from our analysis because these were often found at advanced stages with poor prognosis, and patients were therefore not subjected to further investigations such as colonoscopy. Furthermore, separating analyses of duodenal adenomas from carcinomas may yield important pathogenetic insights on neoplastic initiation and promotion as only a small subset of adenomas progresses to carcinoma.

A possible explanation for the strong association between duodenal adenoma and colorectal neoplasia in this study may be that some patients had undiagnosed FAP. Although attenuated FAP is a possibility, the commoner FAP phenotypes are unlikely given that multiple polyps, if not gross carpeting, of the colonic mucosa is frequently seen. Duodenal adenomas in FAP are also frequently multiple, a feature absent in this patient series. ${ }^{14}{ }^{15}$ Recently, biallelic mutations in the $M Y H$ gene encoding a base excision-repair protein have been described, in which patients develop multiple colorectal neoplasias and occasional associated duodenal adenomas. ${ }^{16}$ It is possible that some of our cases were due to this recessive syndrome. Some of the patients in our series may also have undiagnosed HNPCC, which increases the risk of small intestinal cancer by 25 -fold over the population average. ${ }^{17}$ Of

Table 3 Odds ratios for significant differences between duodenal adenoma patients, endoscoped controls, and the population

\begin{tabular}{lccc}
\hline Colonic neoplasia found & p Value & OR & $95 \% \mathrm{Cl}$ \\
\hline $\begin{array}{l}\text { All colorectal neoplasia } \\
\text { DA } v \text { endoscoped controls }\end{array}$ & 0.03 & 2.5 & $1.1-5.4$ \\
$\begin{array}{l}\text { Cancer or advanced adenoma } \\
\text { DA } v \text { endoscoped controls }\end{array}$ & 0.05 & 2.7 & $1.0-5.2$ \\
$\begin{array}{l}\text { Cancer } \\
\text { DA } v \text { endoscoped controls }\end{array}$ & 0.05 & 3.0 & $1.0-9.1$ \\
$\begin{array}{l}\text { Cancer } \\
\text { DA } v \text { population ( }=34)\end{array}$ & $<0.001$ & \\
\hline DA, patients with duodenal adenomas; OR, odds ratio; $95 \% \mathrm{Cl}, 95 \%$ confidence intervals.
\end{tabular}


Table 4 Indications for colonoscopy in duodenal adenoma patients and endoscoped control patients

\begin{tabular}{lcccc}
\hline Indication & DA (n=34) & $\begin{array}{l}\text { Colorectal neoplasia } \\
\text { found for indication }\end{array}$ & $\begin{array}{l}\text { Endoscoped } \\
\text { controls (n= 102) }\end{array}$ & $\begin{array}{l}\text { Colorectal neoplasia } \\
\text { found for indication }\end{array}$ \\
\hline Anaemia & $13(38 \%)$ & $7(54 \%)$ & $27(26 \%)$ & $12(44 \%)$ \\
Frank bleeding & $2(6 \%)$ & $2(100 \%)$ & $25(25 \%)$ & $9(36 \%)$ \\
$\begin{array}{l}\text { Altered bowel habit } \\
\text { Diarrhoea }\end{array}$ & $2(6 \%)$ & $2(100 \%)$ & $14(14 \%)$ & $4(29 \%)$ \\
Constipation & & $5(5 \%)$ & 0 \\
Abnormal $x$ ray & & $2(2 \%)$ & 0 \\
Weight loss & & $5(5 \%)$ & $2(40 \%)$ \\
Pain & & $3(3 \%)$ & $2(33 \%)$ \\
Family history CRC & $2(6 \%)$ & $1(50 \%)$ & $2(3 \%)$ & $1(50 \%)$ \\
Follow up polyp & $75(44 \%)$ & $7(47 \%)$ & $6(6 \%)$ & $3(50 \%)$ \\
Duodenal adenoma & 34 & $19(56 \%)$ & 102 & $34(33 \%)$ \\
Total & & & \\
\hline DA, duodenal adenoma patients; CRC, colorectal cancer. & &
\end{tabular}

these cancers, approximately one third involve the duodenum. ${ }^{18}$ Indeed, spontaneous mutations can occur in the mismatch repair genes in the absence of a family history of HNPCC associated cancer. Future examination for microsatellite instability and MLH1/MSH2 protein expression in duodenal adenomas in this series would be useful to identify potential undiagnosed HNPCC cases.

Another explanation for the association between duodenal and colonic neoplasia may be that they share common pathogenetic pathways. This may include genetic and/or environmental factors. The data on this issue are currently unclear. For instance, analyses of genetic mutational steps in duodenal and colorectal carcinogenesis show differences in the frequency and site of APC gene mutations ${ }^{19-21}$ while showing some positive correlations for late events, such as

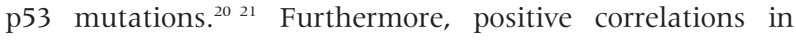
protein and fat consumption have been found between the small intestinal and colorectal carcinoma. ${ }^{9}$

Given that many of the colorectal neoplasias identified in patients with duodenal adenomas were found on investigation for colonic symptoms, is it appropriate to reserve colonoscopy only for those duodenal adenoma patients who have, or develop, symptoms referable to colonic disease? This study found that seven of 15 patients $(47 \%)$ who underwent colonoscopy purely on the basis of the finding of a duodenal adenoma were subsequently found to harbour colorectal neoplasia. Although the number of patients was small, we believe that colonoscopy is indicated for all duodenal adenoma patients. An analogous situation arises when asymptomatic patients found to have left colonic adenomas on sigmoidoscopy are recommended to have complete colonoscopy due to a $30 \%$ incidence of synchronous proximal colonic neoplasia. ${ }^{22-24}$ Importantly, if colonoscopy was automatically recommended in patients with a sporadic duodenal adenoma in this study, one sigmoid cancer would have been discovered three months prior to presenting with rectal bleeding, while another cancer may have been prevented, by detection of an advanced neoplastic lesion five years earlier. Furthermore, as neither specific location nor histology of the duodenal adenoma was useful in determining who would derive greater benefit from colonoscopy, it seems reasonable to recommend colonoscopy for all patients identified with a duodenal adenoma.

In summary, this study confirms a significant association between duodenal adenoma and colorectal adenoma and carcinoma outside of recognised hereditary colorectal cancer syndromes. We therefore recommend colonoscopy in all patients found to have a duodenal adenoma and consider it prudent to maintain ongoing colonoscopic surveillance for these patients, in a similar fashion to those with identified colorectal neoplasia.

\section{ACKNOWLEDGEMENTS}

The authors wish to thank the following for their invaluable assistance: Dr Neville Hoffman for generation, maintenance, and interrogations of the endoscopic database; Drs Bastiaan de Boer and Bruce Latham for extracting histopathology data; and Dr Karen Byth for biostatistics advice.

\section{Authors' affiliations}

M A Murray, H C Ee, Department of Gastroenterology and Hepatology, Sir Charles Gairdner Hospital, Western Australia, Australia

M J Zimmerman, Department of Gastroenterology, Royal Perth Hospital, Western Australia, Australia

\section{REFERENCES}

1 Hochter W, Weingart J, Seib HJ, et al. Duodenal polyps. Incidence, histologic substrate and significance. Dtsch Med Wochenschr 1984; 109:1183-6.

2 Jepsen JM, Persson M, Jakobsen NO, et al. Prospective study of prevalence and endoscopic and histopathologic characteristics of duodenal polyps in patients submitted to upper endoscopy. Scand J Gastroenterology 1994;29:483-7.

3 Sellner F. Investigations on the significance of the adenoma-carcinoma sequence in the small bowel. Cancer 1990;66:702-15.

4 Muto T, Bussey HJR, Morson BC. The evolution of cancer of the colon and rectum. Cancer 1975;36:2251-70.

5 Fearon ER, Vogelstein B. A genetic model for colorectal tumorigenesis. Cell 1990:61:759-67.

6 Perzin KH, Bridge MF. Adenomas of the small intestine: A clinicopathologic review of 51 cases and a study of their relationship to carcinoma. Cancer 1981;48:799-819.

7 Arber N, Neugut Al, Weinstein IB, et al. Molecular genetics of small bowel cancer Cancer Epidemiol Biomarkers Prev 1997;6:745-8.

8 Neugut Al, Santos J. The association between cancers of the small and large bowel. Cancer Epidemiol Biomarkers Prev 1993;2:551-3.

9 Lowenfels AB, Sonni A. Distribution of small bowel tumors. Cancer Lett 1977:3:83-6.

10 Seifert E, Schulte F, Stolte M. Adenoma and carcinoma of the duodenum and papilla of Vater: a clinicopathologic study. Am J Gastroenterol 1992;87:37-42.

11 Atkins WS, Morson BC, Cuzick J. Long-term risk of colorectal cancer after excision of rectosigmoid adenomas. N Engl J Med 1992;326:658-62.

12 Winawer SJ, Fletcher RH, Miller L, et al. Colorectal cancer screening: clinical guidelines and rationale. Gastroenterology 1997; 1 12:594-642.

13 Australian Institute of Health and Welfare (AlHW) and Australasian Association of Cancer Registries (AACR) 2001. Cancer in Australia 1998. Canberra: AlHW (Cancer Series No 17), 2001;AlHW cat No CAN 12 (http://www.aihw.gov.au/publications/can/ca98).

14 Spigelman AD, Talbot IC, Penna C, et al. Evidence for adenoma-carcinoma sequence in the duodenum of patients with familial adenomatous polyposis. $J$ Clin Pathol 1994;47:709-10.

15 Yao T, lida M, Ohsato K, et al. Duodenal lesions in familial polyposis of the colon. Gastroenterology 1977;73:1086-92.

16 Sieber OM, Lipton L, Crabtree M, et al. Multiple colorectal adenomas, classic adenomatous polyposis, and germ-line mutations in MYH. N Engl J Med 2003;348:791-9.

17 Watson P, Lynch HT. Extracolonic cancer in hereditary nonpolyposis colorectal cancer. Cancer 1993;71:677-85.

18 Rodriguez-Bigas MA, Vasen HFA, Lunch HT, et al. Characteristics of small bowel carcinoma in hereditary nonpolyposis colorectal carcinoma. Cancer 1998;83:240-4. 
19 Achille A, Scupoli MT, Magalini AR, et al. APC gene mutations and allelic losses in sporadic ampullary tumours: evidence of genetic difference from tumours associated with familial adenomatous polyposis. Int J Cancer 1996;68:305-12

20 Wheeler JMD Warren BF, Mortensen NJMCC et al. An insight into the genetic pathway of adenocarcinoma of the small intestine. Gut 2002;50:218-23.

21 Arai M, Shimizu S, Imai Y, et al. Mutations of the Ki-ras, p53 and APC genes in adenocarcinomas of the human small intestine. Int $J$ Cancer 1997;70:390-5.
22 Schoen RE, Corle D, Cranston L, et al. Is colonoscopy needed for the nonadvanced adenoma found on sigmoidoscopy? Gastroenterology 1998; 115:533-41.

23 Imperiale TF, Wagner DR, Lin CY, et al. Risk of advanced proximal neoplasms in asymptomatic adults according to the distal colorectal findings. NEngl J Med 2000;343:169-74.

24 Sciallero S, Bonelli L, Aste $\mathrm{H}$, et al. Do patients with rectosigmoid adenomas $5 \mathrm{~mm}$ or less in diameter need total colonoscopy? Gastrointest Endosc 1999;50:314-21

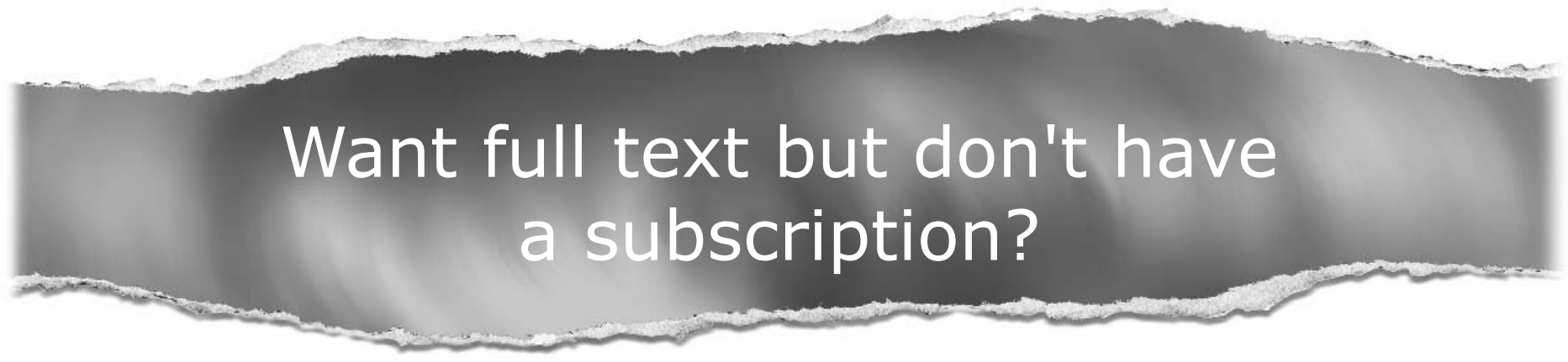

\section{Pay per view}

For just $\$ 8$ you can purchase the full text of individual articles using our secure online ordering service. You will have access to the full text of the relevant article for 48 hours during which time you may

download and print the pdf file for personal use.

www.gutjnl.com 